\title{
Toward brain-computer interface based wheelchair control utilizing tactually-evoked event-related potentials
}

\author{
Tobias Kaufmann ${ }^{*}{ }^{\dagger}$, Andreas Herweg ${ }^{\dagger}$ and Andrea Kübler
}

\begin{abstract}
Background: People with severe disabilities, e.g. due to neurodegenerative disease, depend on technology that allows for accurate wheelchair control. For those who cannot operate a wheelchair with a joystick, brain-computer interfaces $(\mathrm{BCl})$ may offer a valuable option. Technology depending on visual or auditory input may not be feasible as these modalities are dedicated to processing of environmental stimuli (e.g. recognition of obstacles, ambient noise). Herein we thus validated the feasibility of a BCI based on tactually-evoked event-related potentials (ERP) for wheelchair control. Furthermore, we investigated use of a dynamic stopping method to improve speed of the tactile $\mathrm{BCl}$ system.

Methods: Positions of four tactile stimulators represented navigation directions (left thigh: move left; right thigh: move right; abdomen: move forward; lower neck: move backward) and $\mathrm{N}=15$ participants delivered navigation commands by focusing their attention on the desired tactile stimulus in an oddball-paradigm.

Results: Participants navigated a virtual wheelchair through a building and eleven participants successfully completed the task of reaching 4 checkpoints in the building. The virtual wheelchair was equipped with simulated shared-control sensors (collision avoidance), yet these sensors were rarely needed.

Conclusion: We conclude that most participants achieved tactile ERP-BCI control sufficient to reliably operate a wheelchair and dynamic stopping was of high value for tactile ERP classification. Finally, this paper discusses feasibility of tactile ERPs for BCl based wheelchair control.
\end{abstract}

Keywords: Brain-computer interface, Event-related potentials, P300, Tactile, Wheelchair, Dynamic stopping

\section{Background}

Brain-computer interfaces $(\mathrm{BCI})$ allow for direct communication between a person's brain and technical devices without the need for motor control (for review, [1-4]). BCIs thus constitute a promising assistive technology device for people with severe motor impairment, e.g. due to neurodegenerative disease (e.g., [5-10]). Among many different applications, researchers suggested their use for wheelchair control (e.g., [11]), thus rendering BCIs of high value for people with severe paralysis who are not able to control a wheelchair by means of a joystick (e.g., [12]).

\footnotetext{
* Correspondence: tobias.kaufmann@uni-wuerzburg.de

${ }^{\dagger}$ Equal contributors

Department of Psychology I, University of Würzburg, Marcusstr 9-11,

Würzburg 97070, Germany
}

For example, people with intermediate spinal muscle atrophy (SMA, type II) are usually in need of a wheelchair at a young age. With progression of the disease, they may lose control of a wheelchair even by means of a small finger joystick. Control with eye-tracking devices is not feasible, as they obviously need the visual modality for observation of their environment during navigation. Facial muscles may also lose their reliability and are rapidly fatigued in frequent use [13]. With progression of disease, BCIs may become a feasible alternative for wheelchair control.

Among different input signals for BCI control, electroencephalography (EEG) appears viable for wheelchair control due to its high temporal resolution and portability. Most studies on wheelchair control by means of a $\mathrm{BCI}$ investigated sensorimotor rhythms (SMR) as input signal that can be modulated voluntarily by motor imagery $(\mathrm{MI} ;[14,15])$. It 
is possible to discriminate between different imageries or for example between imagery and rest. Each command is referred to as one class, e.g. left hand vs. right hand MI would be referred to as a two-class SMR-BCI paradigm. Different protocols have been suggested for wheelchair (or robot) navigation tasks that either analyze ongoing EEG activity (asynchronous control, i.e. a command can be delivered at any time; e.g., [11,12,16-19]) or analyze EEG activity at a given time window (synchronous control, i.e. a command can be delivered only at a certain time; e.g., [20-22]). The latter require cues that trigger the time windows and display them to the user. Such cues can be presented visually. However, to achieve SMR modulations without occupying the visual channel (i.e. visual cue on a screen), auditory-cued paradigms have been validated (auditory: e.g., [21,23]; auditory + visual: e.g., [20]). Furthermore, feedback can be presented through tactile stimulation units (e.g., [24,25]).

As any error made while controlling a wheelchair may immediately cause damage (or even danger for the patient), wheelchairs may be equipped with shared control systems, i.e. sensors that for example prevent collisions or regulate speed while approaching an object (e.g., [12,16,26-28]). Such shared control systems usually also dedicate parts of the movement control to the wheelchair as BCIs are not yet capable to operate on a full control level as possible with motor control [29]. One reason is, that the number of classes in SMR based BCIs is limited, as discrimination between different MI patterns becomes more difficult with increasing class number, and intensive training may be required [30]. Thus, researchers introduced paradigms that extrapolate different navigation commands from few MI classes only, e.g. translate three MI classes into six different commands [11] or two MI classes into three different commands [20,30].

Such translation, however, may require tasks that are more complex and entail slower rates for communicating commands. Furthermore, a general issue with motor imagery based BCIs is that for many participants SMR-BCIs are inefficacious or display large performance variations across runs [31-35]. However, reliability of BCI commands is particularly necessary for accurate wheelchair control. In a recent evaluation study, severely motor impaired endusers rated reliability of $\mathrm{BCI}$ applications controlled by event-related potentials (ERP) high [10]. ERP-based systems may thus constitute a more reliable alternative to SMR as input signal for wheelchair control, although users cannot actively modulate ERPs for control command generation but need external stimulation. ERP-BCIs make use of a socalled oddball-paradigm, i.e. rare but relevant stimuli are presented within frequent, but irrelevant stimuli. Users focus their attention by counting the rare target stimuli whilst ignoring all other (non-target) stimuli. Target stimuli will evoke more pronounced negative and positive potential fluctuations in the event-related EEG than non-target stimuli (for review on the paradigm, [36]). The most prominent potential in ERP-BCI systems usually is the P300, a positive deflection around 300 milliseconds post-stimulus ([37], its amplitude, shape and latency strongly varies with paradigms and subject-specific conditions; for review, e.g., [38]), which is why ERP-BCIs were often referred to as P300-BCIs (originally by [39]; for comparison of ERPs contributing to ERP-BCI performance [40]; for recent review [36,41-43]). By detecting the elicited ERPs, classification algorithms can identify the intended target selection and translate it into a control command.

Several ERP-based BCI systems for wheelchair (or robot) control have been proposed that differ strongly concerning the amount of control that is left to the user. Rebsamen and colleagues [44] proposed a system, which allowed users to select the targeted destination in a building (e.g. the kitchen) from a visually displayed ERP-BCI matrix. The wheelchair will then autonomously drive to the selected location. This fully transfers navigation control to the smart wheelchair and users can only interfere through selecting a stop mechanism that will terminate the movement. A similar level of control was proposed for control of a humanoid robot [45]. Users selected targeted objects or locations from a series of camera screenshots used as stimuli in an oddballparadigm. The robot then autonomously approached and picked up the object. The advantage of such systems with which users select high-level goals (e.g. a location) while the system performs all low-level operations (steering toward the location) usually lies in its speed and accuracy. However, its performance fully depends on which and how many environmental conditions the device can handle. In addition, users may well prefer to have more process control on their side, as situational goals may change and the goal selection options of the smart wheelchair may not cover all goals.

An ERP-BCI for actual navigation control can easily be implemented by displaying direction arrows in a visual ERP-BCI matrix, i.e. the wheelchair is steered step by step by selecting the upcoming movement direction from a separately displayed matrix [46]. Iturrate and colleagues proposed a more advanced ERP-BCI for navigation control [47]. The authors equipped a wheelchair with a screen that displayed a reconstruction of the real environmental scenario in real time. Target locations were displayed in the reconstruction model and could be selected using an ERP-BCI. Consequently, the system leaves more decisions to the user, yet the actual target locations are computed by the smart wheelchair, i.e. users can only select those target locations that are recognized as possible locations by the detection sensors. This system was recently developed further for control of a telepresence mobile robot [48]. Furthermore, different input signals can be combined for wheelchair control in a hybrid approach (e.g., [49]). Long and colleagues [49] implemented a system that 
controlled direction by means of SMR modulation and speed with a visual ERP-BCI.

Although visually elicited ERPs usually provide best classification accuracies [50] and thus highest information transfer rates compared to other modalities (for review, e.g., [36]), there are several issues with regard to wheelchair control. The same issues apply to BCIs based on steadystate visual evoked potentials (SSVEP, e.g., [51]) (1) Visual stimulation requires a display mounted in the visual field of the user, which is critical for those with severe impairment not able to move the neck for looking past the screen to observe their environment they navigate through. (2) Users cannot observe their environment in the process of target selection, as they need to pay attention to the visual stimulation. (3) Changing light settings may negatively influence the efficacy of BCIs that rely on visual stimulation (e.g. due to bright sun).

In light of these restrictions, Brower and van Erp proposed to tactually elicit ERPs for BCI control [52]. Such tactile BCIs use tactile vibration units (called tactors) placed on participants' body, e.g. on hands and wrists [50], on different positions around the waist [52-54] or on the back of participants [54]. Similar to the visual oddball-paradigm, tactors are stimulated randomly (i.e. they vibrate for a short time) and participants focus their attention on one of the tactors (target) whilst ignoring all others (non-targets). Stimuli will elicit distinct ERPs among which the most prominent is the above described P300 component ([54]; for a thorough investigation of tactually-evoked ERPs in a BCI setting). Brouwer and van Erp [52] investigated how stimulus uncertainty (i.e. the number of stimuli used) and stimulus timing affect classification accuracy and found equal accuracies for two, four and six tactors. For stimulus timing, they found similar parameters feasible as used for visual ERP-BCIs. Thurlings and colleagues [54] found, that placement of tactors significantly affected offline BCI performance in a paradigm that applied tactors for control-display mapping (i.e. mapping between navigation directions and tactor location). A placement that was congruent with the navigation environment provided best results. Recently, a case study reported tactile stimulation feasible for reliable elicitation of ERPs in a patient with classic locked-in syndrome [55]. Results were more robust in the tactile than in the auditory or the visual domain. Our current study is based on these results that established a basis for tactile ERP-BCI based navigation.

In contrast to the above described studies on wheelchair control that use SMRs, SSVEPs or visually-evoked ERPs as input signal, this study investigated feasibility of tactuallyevoked ERPs for wheelchair control. (1) We exposed participants to a virtual environment. Participants steered a virtual wheelchair in real time by selecting one of four tactor locations. This approach allowed us to investigate how more complex (and realistic) scenarios affect user performance. Navigation tasks can be regarded as more complex, as users individually decide on the path they take and as processing of their environment may distract them. (2) Recently, researchers reported great benefit of dynamic stopping methods for visual and auditory BCIs (e.g., [56-60]; for comparison of techniques $[61,62])$. The proposed algorithms stop the stimulation cycle when classification reached sufficient probability for identification of the intended target from the event-related EEG. Thus, they dynamically adjust the number of stimulation cycles based on users' individual brain signals. In this work, we investigated the potential of dynamic stopping on performance and timing in tactile ERP-BCIs. (3) Finally, we evaluated device satisfaction following the user-centered approach $[10,63]$.

\section{Methods}

\section{Participants}

$\mathrm{N}=17$ healthy participants were recruited for this study. We excluded one participant due to incompliance with the experimental protocol and one participant stopped before the end of the experiment. The final sample thus comprised $\mathrm{N}=15$ participants (12 female, mean age: $\mathrm{M}=21.8$ years, $\mathrm{SD}=2.9$, range $18-27$ years). All had normal or correctedto-normal vision and none reported any neurological disorders. All participants were naïve with regard to tactually evoked ERP-BCIs. We conducted the experiment in accordance with standard ethical guidelines as defined by the Declaration of Helsinki (World Medical Association) and the European Council's Convention for the Protection of Human Rights and Dignity of the Human Being with regard to the Application of Biology and Medicine (Convention on Human Rights and Biomedicine). All participants gave written informed consent prior to the study. The study was approved by the ethics committee of the Institute of Psychology at University of Würzburg, Germany.

\section{Equipment and data acquisition}

Eight tactile stimulators, i.e. vibrate transducers ( $\mathrm{C} 2$ tactors; Engineering Acoustic Inc., Casselberry, USA), were grouped into pairs of two and attached to a participant's left thigh (top, toward knee), right thigh (top, toward knee), abdomen (above navel) and lower neck (at the height of C4 to C8) using Velcro ${ }^{\circ}$ belts. Prior to the experiment participants had the opportunity to stimulate all tactors individually, to ensure that they adequately perceived all stimulations. During the experiment, each pair of tactile stimulators constituted one target, i.e. two tactors at close position were stimulated simultaneously. We found that grouping two tactors into one target facilitated participants' recognition of stimuli in a pilot study. Stimulus duration was set to $220 \mathrm{~ms}$ and inter-stimulus interval to $400 \mathrm{~ms}$. Stimulation frequency was $250 \mathrm{~Hz}$. 
EEG was acquired from 16 passive $\mathrm{Ag} / \mathrm{AgCl}$ electrodes at positions Fz, FC1, FC2, C3, Cz, C4, CP1, CP2, P7, P3, Pz, $\mathrm{P} 4, \mathrm{P} 8, \mathrm{O} 1, \mathrm{Oz}$ and $\mathrm{O} 2$ ([5]) with ground and reference being applied to right and left mastoid respectively. Impedance was kept below $5 \mathrm{k} \Omega$. Signals were amplified using a g.USBamp (g.tec Engineering GmbH, Graz, Austria) and recorded at a sampling rate of $512 \mathrm{~Hz}$. Band pass filtering between 0.1 and $60 \mathrm{~Hz}$ and notch filtering between 48 and $52 \mathrm{~Hz}$ were applied online.

\section{Software implementations Tactile stimulation}

We implemented control of the $\mathrm{C} 2$ tactor API in $\mathrm{C}++$ and integrated it into the BCI2000 software (Version 3.0; [64]). We modulated the P3Speller module, usually used for communication of characters (for details on the procedure see [39]), such that flashing of the visual character matrix triggered stimulation of tactor pairs (see section "Equipment and data acquisition"). In a $4 \times 4$ character matrix, flashing of row 1 or column 1 would trigger stimulation of tactor pair 1, row 2 or column 2 would trigger tactor pair 2 , etc. Consequently, a $4 \times 4$ matrix triggers four possible targets (the diagonal). The underlying spelling matrix was invisible to the participants.

\section{Feedback paradigms}

Participants were guided through the calibration and copy task runs (see section "Study design") such that the current target was displayed on a screen, i.e. target positions on the body were presented in a schematic side- and top view. Figure 1A provides a screenshot of the presented display during the calibration phase. The same display was also presented during the copy task runs except that feedback on the outcome of classification was provided in real time. We implemented the paradigms in Python 2.5 (using Pygame 1.9 and PyOpenGL 3.0) and connected them to BCI2000 via user datagram protocol (UDP). Feedback paradigm and BCI2000 were executed on separate computers.

\section{Virtual environment}

We created a 3D-model of a virtual building in Blender 2.6 (Blender Foundation, Amsterdam, Netherlands). It comprised a single floor with four rooms and a corridor. Figure $1 \mathrm{~B}$ displays a top view of the floor plan. We also modeled a wheelchair and several objects (table, checkpoint flags) in Blender and generated corresponding textures with Gimp 2.8 (www.gimp.org, GNU Image Manipulation program). The Panda3d game engine (Version 1.7; Entertainment Technology Center, Pittsburgh, USA) was used to accomplish motion of the wheelchair through the building. Finally, the virtual environment was connected to BCI2000 via UDP. Figure $1 C$ provides a screenshot of the virtual environment. Participants controlled the wheelchair from a third person perspective (view from behind the neck support of the wheelchair). We chose this perspective as from a first person perspective the wheelchair would not have been visible and participants could not have looked around as would be possible in a real wheelchair setting or virtual environment. As the scenario displayed on the screen was restricted to one view, we consequently chose a view from which they could perceive the wheelchair and their environment. In the upper right corner, a top view map provided position tracking to support orientation in the building.

The virtual wheelchair was equipped with collision sensors imitating the behavior of an intelligent wheelchair. The collision system was implemented independent from the one incorporated in Panda3d's game API, as this preset collision system allows for sliding along walls. This would not be feasible for wheelchair control. The wheelchair was thus equipped with collision sensors that would either stop the wheelchair (prevent collision with an object and/or sliding along it) or slow down the wheelchair's speed to enable for more accurate control (e.g. when passing through a door). Figure 1D illustrates the collision zones of the wheelchair. Detection of objects within the forward or backward collision zones immediately stopped all movement in the specific direction and the wheelchair ignored all further commands in this direction until the zone was cleared again. By utilizing generous forward and backward collision zones we ensured that collision free turning is possible after the wheelchair stopped. Detection of objects within the "slow mode" collision zone reduced the movement and turning speed down to $50 \%$ of the original value until the zone was cleared again.

Each time a pair of tactors was classified as target (left, right, forward or backward; section "Equipment and data acquisition") the wheelchair would either move by 1 virtual meter into the desired direction or turn to the requested side by 45 degrees.

We placed four checkpoints in the building. They illustrated the task of moving along a corridor through a door into the office room to approach the desk. The optimal path to fulfill this task comprised 16 commands with no more than 5 commands in between two check-points (see Figure 1B).

\section{Offline and online classification: dynamic stopping and static stopping}

We refer to classification based on data acquired during a calibration run as offline classification, whereas online classification is classification that is performed during ongoing data collection and results in immediate feedback to the user.

During online runs, data were streamed into MATLAB 2010b (The Mathworks Inc., Massachusetts, USA) using Fieldtrip ([65]; http://fieldtrip.fcdonders.nl). Online classification 

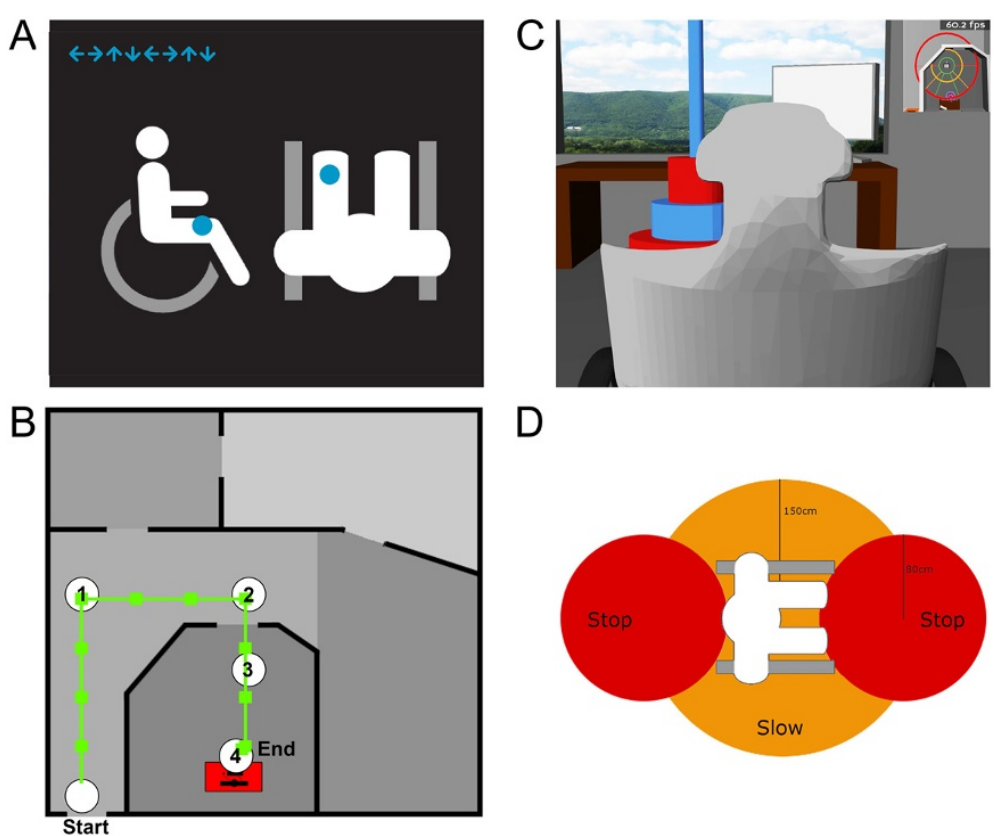

D

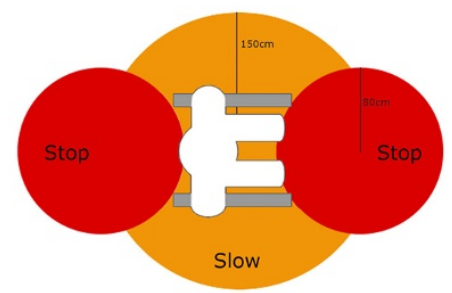

Figure 1 Experimental design. (A) Screenshot of the display presented during the calibration phase. The current target tactor was presented schematically in top and side view. The arrows on the top left indicate the consecutive targets of the run. (B) Top view of the floor plan. Four checkpoints were inserted into the building and participants had to target one after another until reaching a desk at checkpoint 4 . (C) Screenshot of the virtual environment (view from behind the neck support of the wheelchair). The screenshot was taken shortly before reaching the final checkpoint (blue/red stack) close to the desk (left center of the screenshot). In the upper right corner, position tracking was provided for orientation in the building. (D) Collision zones of the wheelchair. When frontally approaching an object (i.e. an object enters the "stop" zone marked in orange), the wheelchair would stop to prevent collision. Furthermore, it would slow down when any objects entered the "slow" zone (green ellipse around the wheelchair).

(stepwise linear discriminant analysis, SWLDA, $800 \mathrm{~ms}$ post-stimulus; as e.g. used in $[39,66,67]$ ) was then performed in MATLAB and results communicated to the feedback applications by means of UDP.

We implemented a dynamic stopping based on a combination and modification of two recently published dynamic stopping methods ([56,57], see introduction). Figure 2 illustrates the decision tree. The tree comprised three basic rules as follows. (1) A minimum number of three sequences were collected for classification. (2) If no decision could be made after gathering a predefined maximum number of sequences (NoS), the most likely target was classified from all gathered sequences of the trial. The maximum number of trials was adjusted for each participant separately based on results from calibration (minimum NoS to reach offline performance estimation of stable $100 \%$ plus two sequences; described in detail in [68]). (3) A dynamic stop could be performed if the most likely target was the same three sequences in a row (modified from [57]) or if a $t$-test with unequal variance performed on so far gathered samples was significant at an alpha level below 10\% (modified from [56]). The alpha level was chosen after pilot testing.

We compared dynamic stopping to the commonly used static stopping, i.e. each trial comprised a fixed number of sequences that were all used for classification. The number of sequences was equal to the maximum number of sequences used in the dynamic stopping run.

\section{Study design}

Before the experiment, participants were instructed and tactors were placed (see section "Equipment and data acquisition"). Participants had the possibility to adjust tactor positions by a few centimeters until they perceived all stimulations equally well. To familiarize the participants with the floor map and with the control principle of the virtual wheelchair, they used a keyboard to move the wheelchair through the virtual environment during EEG preparation.

The actual experiment consisted of one calibration run (predefined task; data is used to compute classifier weights), two copy tasks (predefined task; used to evaluate classifier performance online) and finally the main goal of the study, i.e. one task aiming at navigation through the virtual building. Duration of calibration was $10 \mathrm{~min}$. Duration of copy and navigation tasks were participant specific depending on their performance (see section "Results"). One calibration trial comprised 15 stimulation sequences per tactor pair, i.e. each tactor pair vibrated 30 times (one sequence corresponding to four row and four column flashes in the visual matrix; see section "Software implementations - Tactile stimulation"). Calibration was performed with eight trials (each tactor pair was twice the target). If offline 


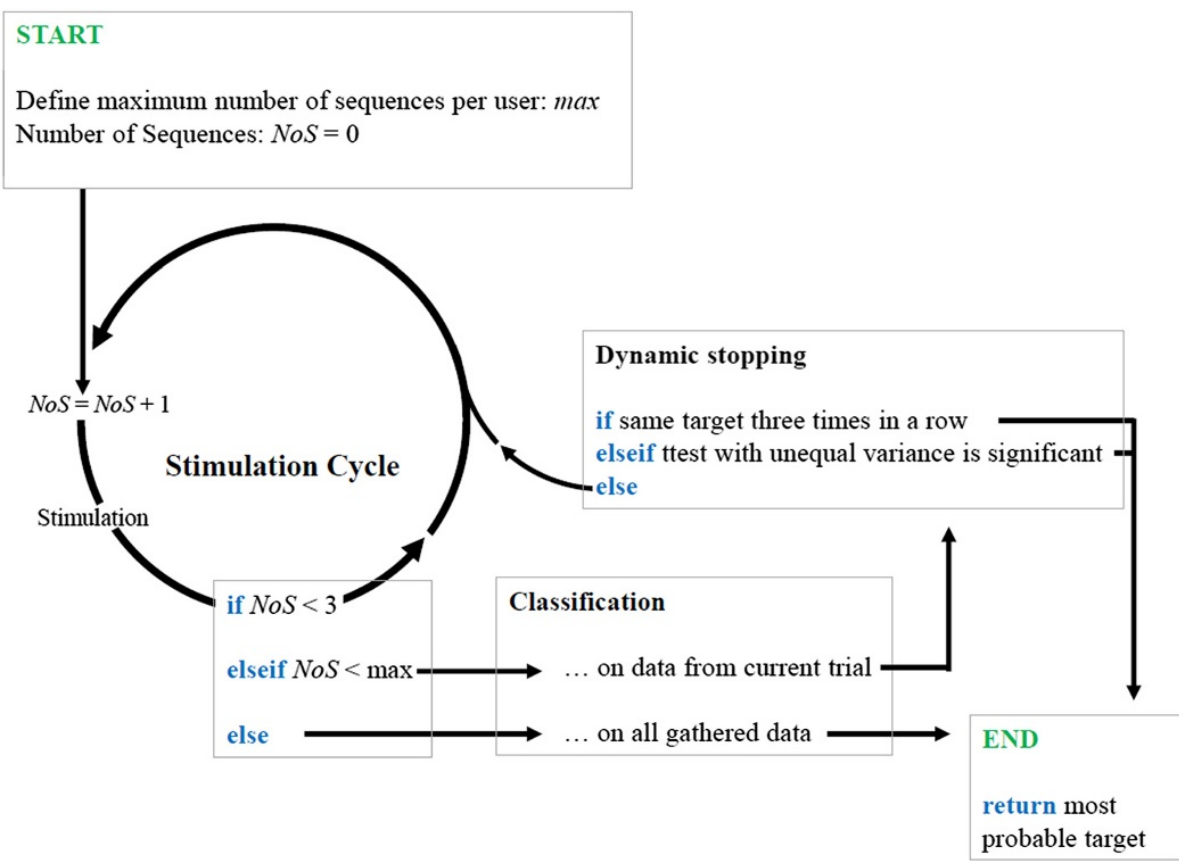

Figure 2 Decision flow chart of the dynamic stopping method.

analysis revealed a performance below $100 \%$ after these eight trials (when including all sequences into classification), we repeated calibration once. After calibration, participants performed two copy task runs. One copy task run included static number of sequences, i.e. each trial comprised a maximum number of sequences before classification. A second copy task run introduced the abovedescribed dynamic stopping method. This allowed for within comparison of performance achieved with and without dynamic stopping. During both copy tasks, immediate feedback on classification outcome was provided to the participants. As for the calibration run, each tactor pair was twice the target, resulting in eight trials per copy task run. Participants then moved on to control of a virtual wheelchair and tried to navigate along the predefined route (see section "Software implementations - Virtual environment"). When reaching one of the four checkpoints, they took a break of approximately one minute before moving on (the $\mathrm{BCI}$ was manually switched off during this time by the experimenter). The number of trials during navigation varied dependent on the participants' performance. In the optimal path (Figure 1B) selection of the "move forward" command was required most frequently. However, as errors had to be corrected, the number of required commands per navigation direction differed between participants.

\section{Offline data processing of ERPs}

EEG data were filtered between 0.1 and $30 \mathrm{~Hz}$ (FIR equiripple) and divided into segments of $800 \mathrm{~ms}$ poststimulus. Determination between targets and non-targets was quantified by computing $R^{2}$ values. For computing the grand average of $\mathrm{R}^{2}$ values we Z-transformed (Fisher's Z) the square root of the determination values for each participant and electrode, averaged across participants and finally retransformed and squared these grand averages.

\section{Analysis of system performance}

In the virtual environment, performance estimation is difficult, as different paths may be feasible for reaching the checkpoints. For example, after an error participants may either steer back by one step or take a different path to approach the next checkpoint. Thus, we asked participants to report during the breaks whether or not the selected targets were the desired targets and performance was computed based on their reports. To control for false reporting, we manually went through each decision and decided if it was goal-oriented. Finally, we aligned these two analyses. Except for two selections, these decisions were similar to the subjects reports (265 selections in total; from the two selections one would slightly increase performance estimate, one would slightly decrease performance estimate). Therefore, we consider adequate to estimate performance based on subjects reports.

The impact of shared control was determined from the number of collisions and the number of times when sensors for slowing down speed were active. Furthermore, we computed the time required for delivering commands from the duration of stimulus and inter-stimulus intervals. Classification time, wheelchair movement duration and duration of the breaks the participants took at each 
checkpoint were not taken into account. Thus, the reported time is system independent and includes only the mandatory time needed for stimulation.

Furthermore, following the user-centered approach we validated the system based on user reports. Participants rated their confidence with tactile ERP-BCI based wheelchair control with forced choice questionnaires. The questions covered learnability, strain, level of control, speed of the system and participants' trust in the used BCI technology [10].

\section{Statistical analysis}

We checked data of achieved BCI performance for normal distribution using Lilliefors - Kolmogorov Smirnov tests. Due to non-normal distributions, we performed pairwise testing with the Mann-Whitney $U$ test. Bonferroni correction to $5 \%$ alpha levels is indicated. Statistical analysis was performed in Matlab 2010b.

\section{Results}

Five participants repeated calibration once due to insufficient offline performance estimates after the first calibration run. Figure 3 displays offline classification performance: $\mathrm{N}=14$ of 15 participants achieved offline classification accuracy of $100 \%$. Their average number of sequences required to reach Stable 100\% offline accuracy (i.e. retaining $100 \%$ performance when adding further sequences) was $\mathrm{M}=4.9(\mathrm{~N}=14, \mathrm{SD}=1.8$, range: $2-8)$. This would correspond to an average time of $\mathrm{M}=24.3 \mathrm{~s}$ per command. Offline performance for participant 15 was estimated Stable $87.5 \%$ with eight sequences, but did not further improve when calibrating on all sequences.

\section{Dynamic vs. static stopping}

We validated tactile stimulation for ERP elicitation online in two copy tasks. Participants gained overall high accuracy levels in both tasks (see Figure 4A). Average accuracy with static number of sequences was $\mathrm{M}=90.8 \% \quad(\mathrm{SD}=13.7$, range $62-100 \%$ ) and nine of 15 participants performed without errors. The time needed to fulfill the task with static stopping ranged from 4.2 to $8.2 \mathrm{~min}(\mathrm{M}=6.1$, $\mathrm{SD}=1.2 \mathrm{~min}$ ), whereas the time needed to fulfill the task with dynamic stopping ranged from 2.6 to $5.4 \mathrm{~min}$ $(\mathrm{M}=3.7, \mathrm{SD}=1.0)$. Performance did not significantly decrease when introducing dynamic stopping $(\mathrm{N}=15$, $\mathrm{Z}=0.70, \mathrm{p}=.48 ; \mathrm{M}=84.2 \%, \mathrm{SD}=23.4$ ), i.e. most participants maintained the performance level achieved with static number of sequences. However, performance for two participants (participant 6 and 15) severely decreased for participant 15 even to chance level (25\%). Furthermore, we investigated if errors were equally distributed across targets. The total amount of errors did not differ between the targets (left: 10\% errors of all left target selections; right: 11.7\%; forward: $13.3 \%$; back: $15 \%$; $\mathrm{N}=15, \mathrm{H}(3)=0.97$, $\mathrm{p}=.81$, Bonferroni adjusted alpha level: $\alpha=.0083$ ).

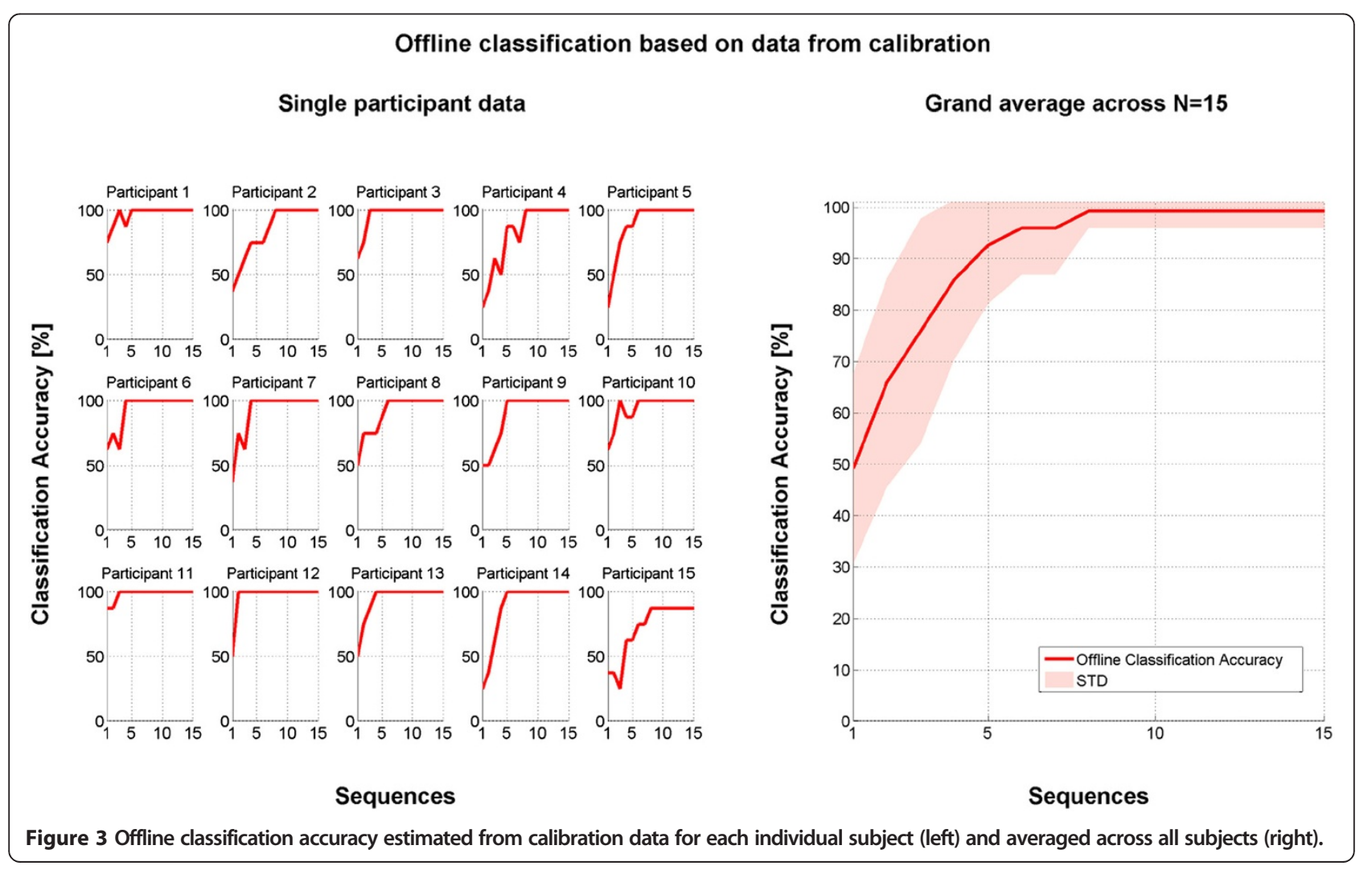




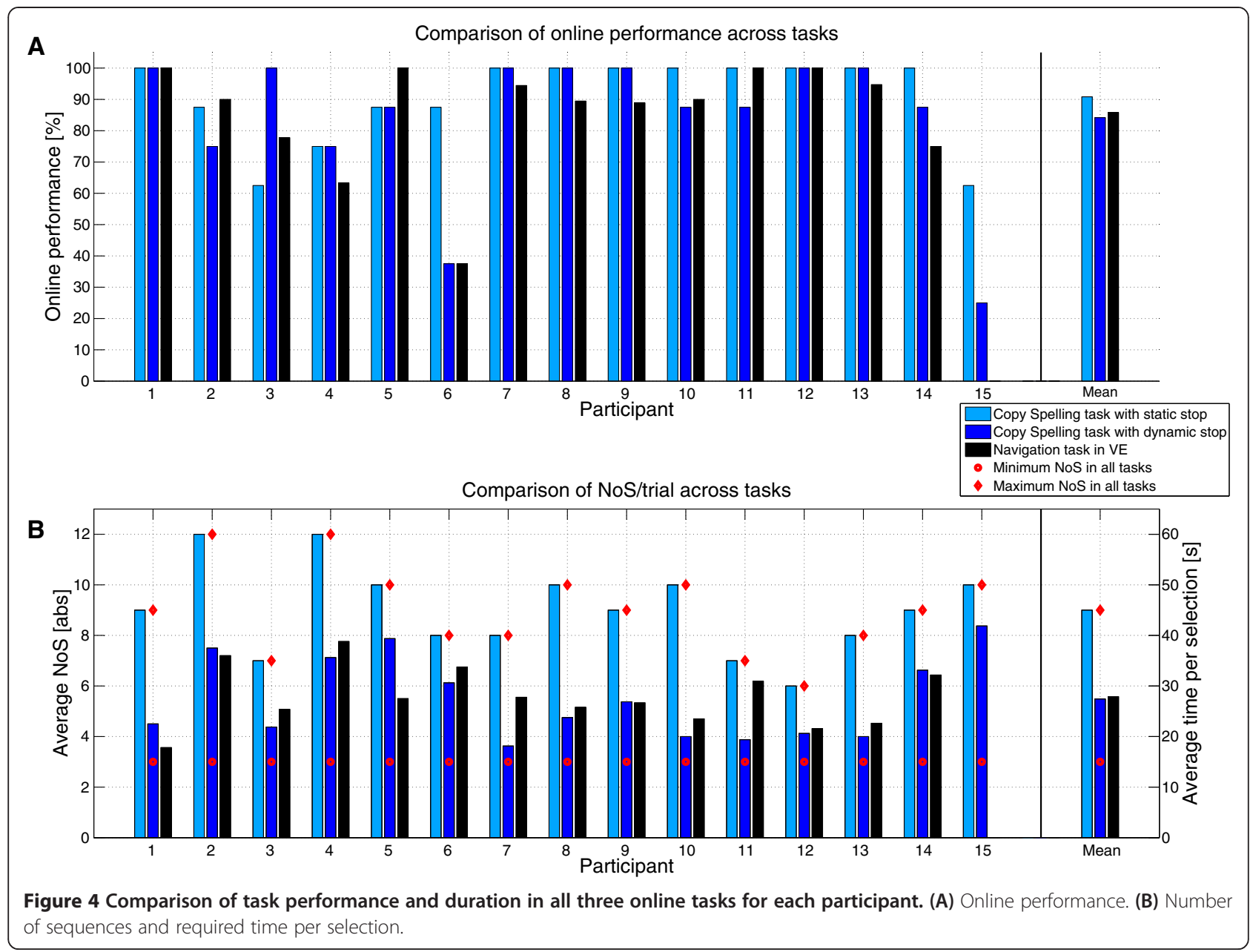

Figure $4 \mathrm{~B}$ depicts the average number of sequences needed to deliver a command. In line with previous reports, the number of sequences significantly decreased in the dynamic stopping copy task $(\mathrm{N}=15, \mathrm{Z}=3.81, \mathrm{p}<.001)$. Consequently participants on average needed $M=27.2$ seconds per selection as compared to $M=44.6$ seconds in the task with static number of sequences.

\section{Wheelchair navigation}

Participant 15 did not perform the navigation task as the performance decreased to chance level when using dynamic stopping in the copy task (section "Results Dynamic vs. static stopping"). Thus, only $\mathrm{N}=14$ of 15 participants performed the navigation task through the virtual building. For each participant, Figure 5 illustrates the path along which they steered the virtual wheelchair. Importantly, $\mathrm{N}=11$ participants reached the targeted desk at checkpoint 4 and four participants made no error. Although the navigation task can be regarded as more complex than a simple copy task, performance did not significantly decrease in the virtual environment $(N=14$,
$\mathrm{Z}=0.33, \mathrm{p}=.74)$. Average accuracy was $\mathrm{M}=85.8 \%$ $(\mathrm{SD}=17.6$, range $37.5-100 \%)$ with a mean of $\mathrm{M}=5.58$ sequences. Three participants, however, could not successfully finish the task and performed the experiment only until they communicated to prefer canceling. Two of them at least managed to pass the corridor before quitting whereas participant 6 again had almost no control (due to dynamic stopping, see section "Results - Dynamic vs. static stopping") and thus canceled the experiment early.

In contrast to the copy tasks that involved no correction of errors, wrong selections in the virtual environment had a direct impact for the further navigation task, i.e. errors had to be corrected. Alike intelligent wheelchairs proposed in robotics research, the virtual wheelchair was thus equipped with simulated shared control sensors. Most participants $(\mathrm{N}=8)$ did not navigate into any situation where these sensors were needed. Collision was prevented once for $\mathrm{N}=4$ participants, twice for participant 3 and five times for participant 14. Sensors for slowing down speed of the wheelchair were active for two participants when passing the door to the office room. Hence, they managed 


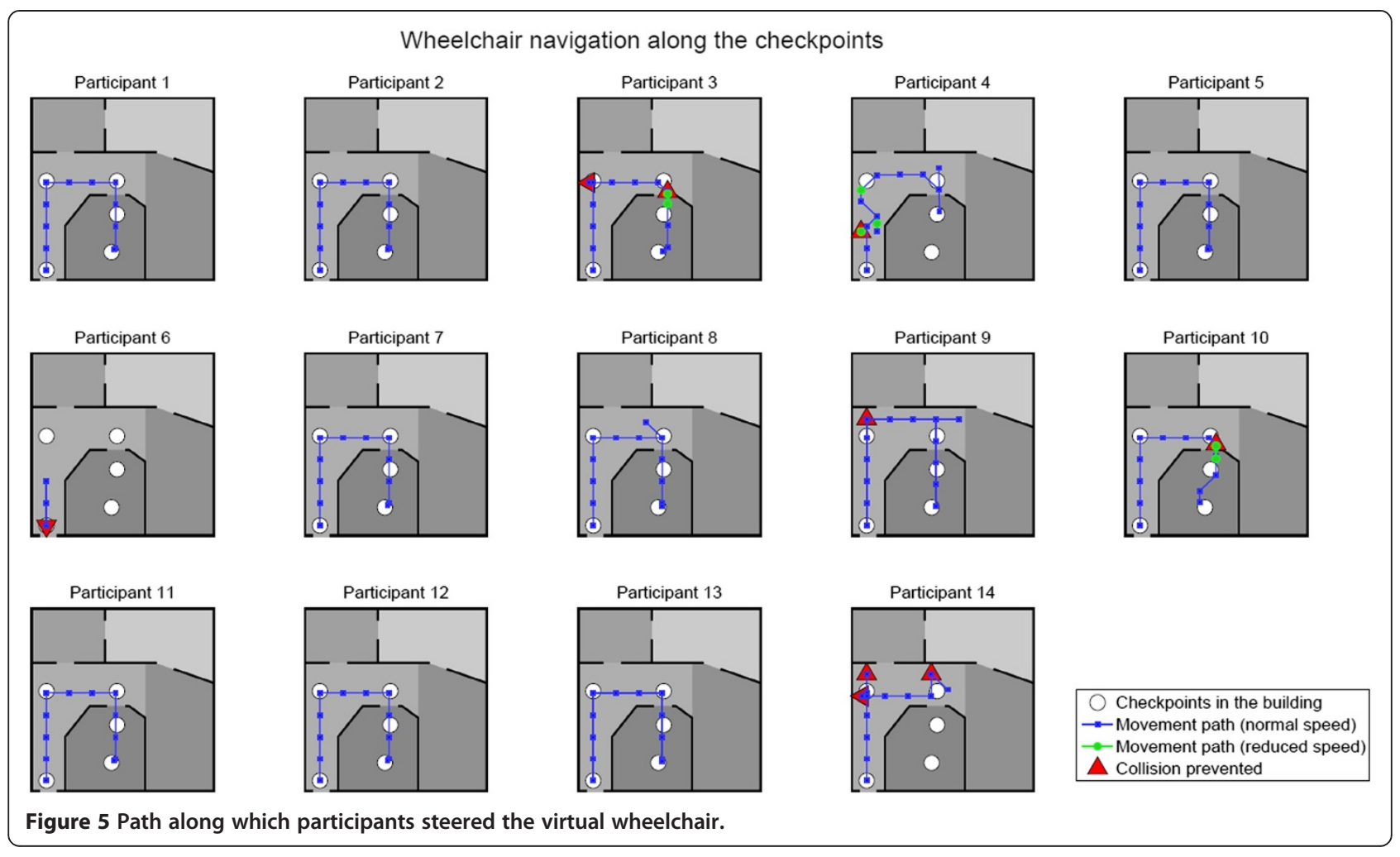

to enter the room and reach the checkpoint. For participant 4 these sensors were activated three times when passing close to a wall, but did not have an effect on the navigation, i.e. they were instantly turned off again with the next movement of the wheelchair (see Figure 5). Table 1 summarizes participants' individual navigation task performances and task durations.

\section{ERP differences in target vs. non-target trials}

During stimulus duration, tactile stimulation of non-target positions also evokes an event-related response as participants directly perceive all stimuli on the body and cannot easily ignore them. Yet after around $300 \mathrm{~ms}$, target and non-target signals diverge. Target stimulation elicits a P300, whereas non-target stimuli often entail a negative ERP in the period between 300 and $500 \mathrm{~ms}$ post-stimulus. ERP responses differed considerably between participants, yet for all of them discrimination between target and nontarget stimuli was possible (see Figure 6). Figure 7 provides a topographical map of the grand-averaged ERPs across all participants based on calibration data.

We further computed the determination coefficients to investigate which features contribute most to classification. As depicted in Figure 8, the centro-parietal electrodes contributed most to discrimination between targets and non-targets. Determination coefficients were highest between 400 and 500 ms, i.e. in the time window of the tactile P300.

\section{Subjective validation with questionnaires}

We further explored system performance using forced choice questionnaires with the four choices "I do not agree at all", "I do not really agree", "I mostly agree", "I fully agree". Table 2 depicts the results. All participants were confident with learning how to control the wheelchair and - except participants 6 and 14 - with reliability of control. As expected, responses to questions on learnability and reliability depended on participants' task performance. With regard to strain and speed participants' answers were independent of their actual performance (Kendalls Tau $\tau=.06, \mathrm{p}=.86$ ). For example, participant 5 who did not perform any error in the virtual environment stated that control was too demanding.

\section{Discussion}

Tactile ERPs for $\mathrm{BCI}$ based wheelchair control

We exposed participants to a virtual environment and asked them to navigate a virtual wheelchair by means of a tactually evoked event-related potential based BCI. Our results are promising in that most of the participants reached the final checkpoint and that only few participants needed shared control. 
Table 1 Summary of participants' individual performances in the wheelchair navigation task

\begin{tabular}{|c|c|c|c|c|c|c|c|c|}
\hline Participant & $\begin{array}{l}\text { Final checkpoint } \\
\text { reached }\end{array}$ & $\begin{array}{l}\text { Time needed [min] } \\
\text { (b.c. = before canceling) }\end{array}$ & $\begin{array}{l}\text { Accuracy } \\
\text { (sensitivity) [\%] }\end{array}$ & Specificity [\%] & $\begin{array}{l}\text { Average time } \\
\text { needed per } \\
\text { selection [s] }\end{array}$ & $\begin{array}{l}\text { Average number of } \\
\text { sequences per } \\
\text { selection [abs] }\end{array}$ & $\begin{array}{l}\text { Collision sensors } \\
\text { needed [abs] }\end{array}$ & $\begin{array}{l}\text { Sensors for slowing the } \\
\text { wheelchair needed [abs] }\end{array}$ \\
\hline 1 & $x$ & 8.8 & 100.0 & 100.0 & 17.7 & 3.6 & - & - \\
\hline 2 & $x$ & 20.8 & 90.0 & 96.7 & 35.7 & 7.2 & - & - \\
\hline 3 & $x$ & 21.0 & 77.8 & 92.6 & 25.2 & 5.1 & 2 & 1 \\
\hline 4 & - & 36.0 b.c. & 63.3 & 88.5 & 38.5 & 7.8 & 1 & 3 \\
\hline 5 & $x$ & 12.8 & 100.0 & 100.0 & 27.3 & 5.5 & - & - \\
\hline 6 & - & 7.8 b.c. & 37.5 & 79.2 & 33.5 & 6.8 & 1 & - \\
\hline 7 & $x$ & 15.0 & 94.4 & 98.2 & 27.6 & 5.6 & - & - \\
\hline 8 & $x$ & 14.4 & 89.5 & 96.5 & 25.6 & 5.2 & - & - \\
\hline 9 & $x$ & 14.3 & 88.9 & 96.3 & 26.6 & 5.3 & 1 & - \\
\hline 10 & $x$ & 14.9 & 90.0 & 96.7 & 23.3 & 4.7 & 1 & 1 \\
\hline 11 & $x$ & 14.3 & 100.0 & 100.0 & 30.7 & 6.2 & - & - \\
\hline 12 & $x$ & 10.3 & 100.0 & 100.0 & 21.4 & 4.3 & - & - \\
\hline 13 & $x$ & 12.7 & 94.7 & 98.2 & 22.5 & 4.5 & - & - \\
\hline \multirow[t]{2}{*}{14} & - & 22.7 b.c. & 75.0 & 92.8 & 31.9 & 6.4 & 5 & - \\
\hline & Total: $N=11$ & Mean: 14.5 (excl. those who canceled) & Mean: 85.8 & Mean: 95.4 & Mean: 27.7 & Mean: 5.6 & Total: $11(\mathrm{~N}=6)$ & Total: $5(\mathrm{~N}=3)$ \\
\hline
\end{tabular}




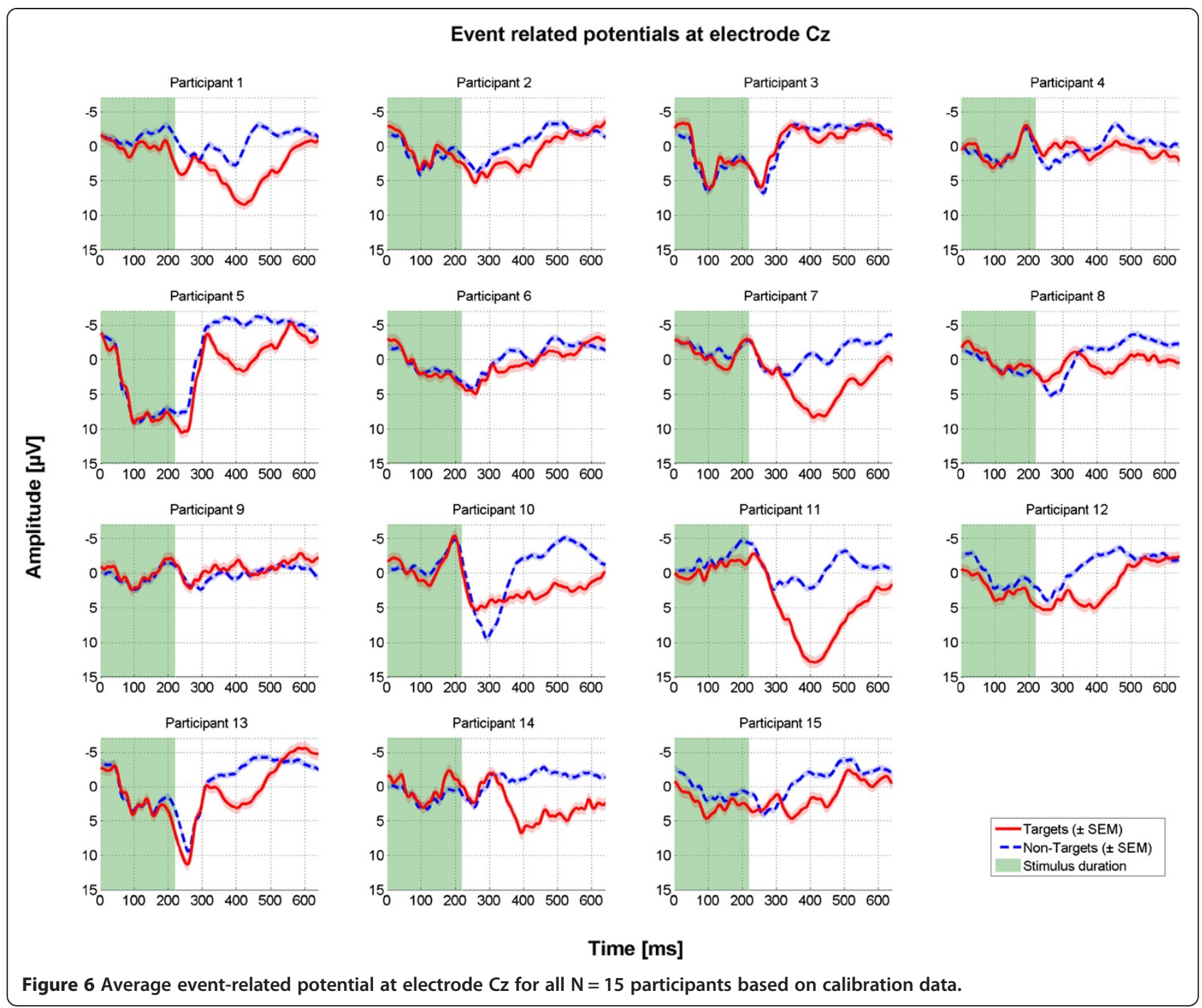

Importantly, $\mathrm{N}=14$ of 15 participants reached $100 \%$ offline classification accuracy and one further participant had an offline accuracy level of $87.5 \%$. In all three online tasks, performance of $\mathrm{N}=11$ participants remained above $70 \%$. For two further participants performance may have remained high (participant 6) or at least medium (participant 15) if we would not have switched to the dynamic stopping method. Tactile ERP-BCIs may thus offer a valuable alternative to motor imagery based $\mathrm{BCIs}$ considering the findings that many SMR-BCI users do not gain sufficiently reliable SMR control [31-35]. Also, SMR-BCIs usually require a longer calibration phase than ERP-BCIs and intensive user training may be necessary to achieve a good level of control, specifically in people with neurodegenerative disease [8]. However, performance varied considerably between participants implying the need for testing larger groups for generalization of results, which is hardly ever the case in studies that use BCI for wheelchair control (e.g., $\mathrm{N}=2$ in $[11,12,16,30,46] ; \mathrm{N}=3$ in [69]; $\mathrm{N}=5$ in $[20,44,47,48]$; and $N=6$ in [22]). Furthermore, often healthy users with prior $\mathrm{BCI}$ experience were selected thereby also hampering generalization of results (e.g., [30,70]). Since all our participants were naïve with regard to tactile ERPBCIs, we speculate that a studious learning of tactile perception (in particular learning to ignore irrelevant tactile stimulations) may further enhance their performance. Furthermore, rebuilding classifiers based on more data input may increase performance, as the short calibration performed at the beginning of the experiment may not be sufficient.

Consequently, in case more data would further enhance classifier accuracy, generic models could be of high value to shorten calibration time (i.e. building a classifier based on data from a large pool of participants; e.g., $[71,72])$. Also, such models may increase performance of those participants who do not achieve accurate 


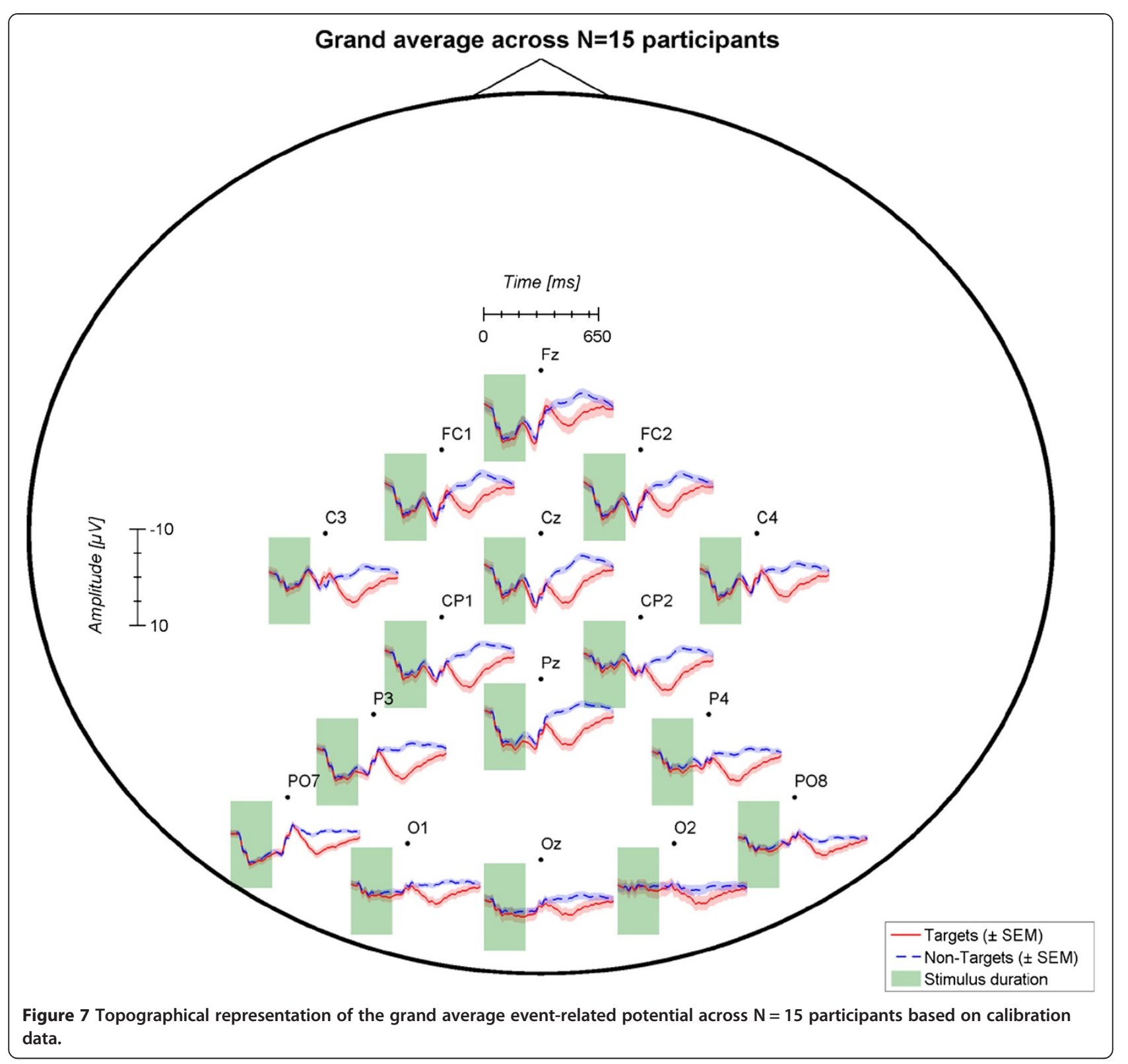

control with their individual classifier [73]. However, our results show large inter-individual differences of the ERPs elicited post-stimulus. In line with previous reports (e.g., [54]) the tactually-evoked P300 peaked at central electrodes with an average latency around 400-500 ms. Centro-parietal electrodes contributed most to classification accuracy. Considering the varying ERP responses across participants, recording from more electrode sites could further enhance subject-specific ERP detection and facilitate investigation of generic models.

Our study design built on prior work on tactile ERP elicitation. Brouwer and van Erp [52] found no performance difference with regard to a number of two, four or six tactile stimulators. We thus implemented a system based on four tactors representing direction control units. Thurlings and colleagues [54] investigated how congruent tactor positioning affects task performance. They positioned a monitor vertically or horizontally in front of participants. A control display mapping was realized with tactors positioned either congruent with monitor angle (i.e. horizontal tactor positions around the waist for horizontal monitor placement and vertical tactor positions on the participants' back in the case of vertical monitor placement) or incongruent (i.e. horizontal tactor positions around the waist and vertical monitor placement). The authors demonstrated that a congruent setup yielded increased P300 amplitudes and thus increased estimated BCI performance. Therefore, in our 


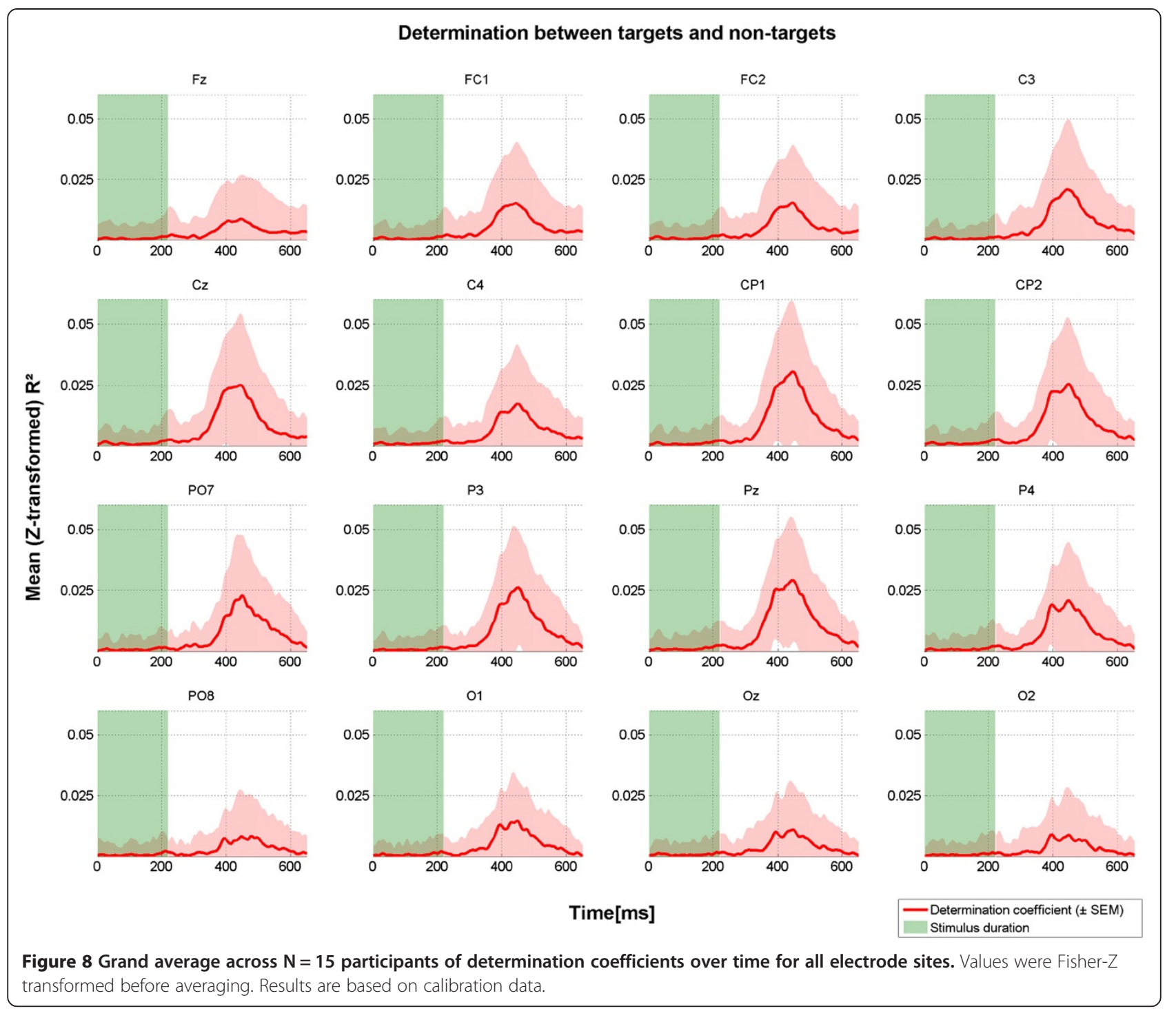

study we aligned tactor placement with movement directions. With regards to stimulus timing we opted for an on-time of $220 \mathrm{~ms}$ and an off-time of $400 \mathrm{~ms}$, i.e. a similar timing than the baseline condition from Brouwer and van Erp [52] in experiments 1 and 2 (188 ms on-time, $367 \mathrm{~ms}$ off-time). The authors suggested matching on- and offtimes and found this condition to enhance bit-rate while maintaining the performance level. Such adjustment may thus also be feasible for our proposed system. However, due to the increased probability of ERP overlap when reducing off-times, we chose the longer duration.

In contrast to Brouwer and colleagues [52], who chose only the front tactor as target, our calibration and online copy tasks comprised equally often all tactors as target.

Table 2 Questionnaires on satisfaction with the tactile ERP-BCI based wheelchair control

\begin{tabular}{|c|c|c|c|c|}
\hline Question & $\begin{array}{l}\text { I do not } \\
\text { agree at all [\%] }\end{array}$ & $\begin{array}{l}\text { I do not really } \\
\text { agree }[\%]\end{array}$ & $\begin{array}{l}\text { I mostly } \\
\text { agree [\%] }\end{array}$ & $\begin{array}{l}\text { I fully } \\
\text { agree [\%] }\end{array}$ \\
\hline Control of the wheelchair was quickly learnable & 0.0 & 0.0 & 71.4 & 28.6 \\
\hline The wheelchair correctly recognized the delivered commands & 0.0 & 14.3 & 71.4 & 14.3 \\
\hline I always had full control over the wheelchair & 14.3 & 0.0 & 71.4 & 14.3 \\
\hline Control of the wheelchair was too demanding & 50.0 & 21.4 & 28.6 & 0.0 \\
\hline Control of the wheelchair was too slow & 0.0 & 64.3 & 35.7 & 0.0 \\
\hline
\end{tabular}


Our results thus account for perception differences or attention difficulties between different body locations. Some participants may for example perceive the front target (close to the navel) stronger than the back target. In our study participants performed equally well on selection of tactors, i.e. in total participants did not perform significantly more errors on any of the targets than on others. Especially in light of a BCI with manifold selection options (realized placing many tactors on the body), it is inherently important to adjust tactor locations according to users' reports so that they perceive all targets (approximately) equally well.

In line with previous reports from visual and auditory ERP-BCIs (e.g., [56-60]; for comparison of techniques [62]), dynamic stopping was of high value also for tactile ERP-BCIs. Participants greatly benefited in terms of time needed to deliver commands, thereby increasing speed of the system. Importantly, the reduced number of sequences in the dynamic stopping copy task did not affect performance (no significant difference between static and dynamic stopping copy task performance) except for two participants who displayed a strong performance drop during dynamic stopping. Hence, these participants did not benefit from dynamic stopping. From the offline classification results as well as from task performance in the copy task with static stopping we assume that participant 6 may have successfully performed the navigation task when using a static number of sequences. As participant 15 did not perform a navigation task, we do not know whether the drop in performance was due to bad performance in one run or due to dynamic stopping. In a comparison of dynamic stopping methods, Schreuder and colleagues [61] reported that some methods decrease performance of participants with less discriminative data. Considering the fact that offline classification performance of participant 15 displayed aggravated discriminability compared to other participants' data, the performance drop may be attributed to dynamic stopping. For all participants, user specific parameter adjustment (as performed by e.g., [56]) could have further increased performance of the dynamic stopping method, especially in the case of those two participants. This may have prevented the algorithm from stopping too early although classification of the target was not sufficient.

Validation of the system based on questionnaires revealed that tactile ERP-BCI based wheelchair control is quickly learnable by naïve participants. Device satisfaction regarding reliability and control was mostly positive. However, evaluation results for demand of control and speed of the system varied and were independent of users' performances. To better estimate these aspects, longer navigation tasks will be needed. On the one hand, learning to perceive stimuli may positively affect the demands for the user, on the other hand long navigation tasks may further increase demands on attention. Users of such systems in daily life navigation tasks may judge speed of the system more critically.

\section{Limitations and future experimentation}

This study explored feasibility of the proposed BCI system in healthy users. We assessed user confidence with forced choice questionnaires to identify remaining issues and how they depend on task performance. However, validation may strongly vary with users' health and with their actual dependence on the technology. Further research must investigate use of tactile ERP-BCIs by the actual target population. In the process of user-centered BCI development, potential end-users with severe motor impairment should be integrated into the design process at an early stage, so that research can specifically account for their needs and requirements $([6,10,63,74-76])$. Furthermore, the effect of proposed improvements may well be larger in patients as compared to healthy participants (as recently found for a modification of visual ERP-BCIs; [7]). In particular, we suggest including patients with SMA type II who we consider a potential target group for use of BCI based wheelchair control. With progression of disease, they usually lose the ability to control a wheelchair with a joystick. Eye-tracking devices would occupy the visual channel needed for observation of their environment and devices based on facial muscles may be too fatiguing. Progression of the disease is usually slower than for example for patients with amyotrophic lateral sclerosis, which renders it more feasible to learn device control when needed. ChelioutHeraut and colleagues [77] reported abnormalities of somatosensory-evoked potentials in a sample of SMA children (type I and II). Yet, these abnormalities occurred far less frequent in SMA type II than in SMA type I. As somatosensory-evoked potential abnormalities were more pronounced in the lower limbs, the proposed tactor positions may not be feasible and thus adjusted individually. The same issue may apply to other types of diseases or injuries, e.g. in the case of spinal cord injury tactile perception on the legs is usually lost. Thus, in all cases, the system requires individually-tailored adjustments based on the sensory perception capabilities of patients.

Generalization of results may be limited with regard to the complexity of the navigation task performed in this study. The path did not require users to select all direction options. From the results of the copy-task, however, it appears unlikely that more errors would have occurred for a different path. Yet, future testing of the system should be performed with several different tasks over a longer period of time. In addition, a vivid environment, in which users need to react to changing settings, could provide useful insights in feasibility of tactual ERP-BCI 
systems under such, more realistic conditions. Finally, generalization may be limited as the third person perspective and the position tracking used in this study may have positively influenced navigation ability, e.g. estimation of distances. However, in a virtual environment it may be more difficult to estimate distances than in a real world setting. Thus, the benefit of position tracking and perspective may be negligible as compared to the benefit of navigating in a real environment.

However, in its current state the system bears some major drawbacks. (1) Some users reported that focusing on tactile stimulation was too demanding in a long navigation task. Thus, stimulation should be enhanced so that users perceive stimuli better. Furthermore, training in several sessions could be conducted to decrease users' workload. Halder and colleagues recently demonstrated, that performance with an auditory ERP-BCI can be improved with training [78]. Zickler and colleagues [10] demonstrated for visual ERP-BCIs that subjective workload of a naïve, severely motor impaired, potential enduser could be strongly decreased the more sessions were conducted, i.e. in his first session he rated workload rather high (49 of 100 on a linear scale) but decreased his rating to 15 in the last session. (2) The average time to deliver a command was roughly 28 seconds, ranging from 17.8 to almost 38.8 seconds. For effective wheelchair control, speed should be further enhanced, e.g. by implementing other dynamic stopping techniques or by increasing the signal-to-noise ratio of the recorded ERPs $[7,79]$. As already addressed above, decreasing the offtime parameter of the system may also enhance speed. (3) The herein tested system is synchronous and not able to detect if a user wants to deliver a navigation command or perform any other task. For example, users may want to interrupt navigation and perform navigationindependent actions (e.g. communicating, reading, observing). It is thus inherently necessary to implement an asynchronous system that will account for such situations [80-82]. (4) Finally, we did not implement an option that rapidly allows for stopping the wheelchair. Once users delivered a movement command, they would hand over full control to the wheelchair, i.e. only its sensors could stop the wheelchair in case of an obstacle. Currently, if they delivered a wrong command, the wheelchair would still perform the action if the requested movement would not interfere with navigation barriers. Implementation of such correction method could be based on residual muscle activity or on other BCI signals in a hybrid approach (e.g., [49,83-86]). This would possibly further reduce the amount of times, when shared control is necessary for intervention. However, already in our experimental setting, participants rarely needed shared control sensors and most of them had full control on the user side.

\section{Conclusion}

We explored tactile ERP-BCI based online wheelchair control in a virtual environment. Participants overall gained high accuracy levels in copy tasks and when navigating through the virtual environment. Importantly, 11 participants finished the requested task, i.e. successfully navigated along four checkpoints. Most participants did not require shared control sensors. In conclusion, our results prove tactile ERP-BCIs feasible for wheelchair control. Yet we discovered and discussed a number of issues to be addressed and solved in future research. Most importantly, data have to be collected with the targeted patient group in the iterative process of user-centered $\mathrm{BCI}$ development.

\section{Competing interests}

The authors declare that they have no competing interests.

\section{Authors' contributions}

TK and AK designed the study. TK and AH programmed and tested the setup. AH collected the data. TK and AH analyzed the data. TK, AH and AK discussed the results. TK drafted the manuscript. AH and AK revised the manuscript. All gave their approval to the final version to be published.

\section{Acknowledgments}

This work was supported by the European ICT Programme Project FP7-224631 (TOBI). This paper only reflects the authors' views and funding agencies are not liable for any use that may be made of the information contained herein. Open access publication was funded by the German Research Foundation (DFG) and the University of Würzburg in the funding programme Open Access Publishing.

Received: 13 March 2013 Accepted: 23 December 2013

Published: 16 January 2014

\section{References}

1. Birbaumer N, Cohen LG: Brain-computer interfaces: communication and restoration of movement in paralysis. J Physiol 2007, 579:621-636.

2. Kübler A, Kotchoubey B, Kaiser J, Wolpaw JR, Birbaumer N: Brain-computer communication: unlocking the locked in. Psychol Bull 2001, 127:358-375.

3. Wolpaw J, Wolpaw EW: Brain-Computer Interfaces: Principles and Practice. New York: Oxford University Press; 2012.

4. Wolpaw JR, Birbaumer N, McFarland DJ, Pfurtscheller G, Vaughan TM: Brain-computer interfaces for communication and control. Clin Neurophysiol 2002, 113:767-791.

5. Hoffmann U, Vesin J-M, Ebrahimi T, Diserens K: An efficient P300-based brain-computer interface for disabled subjects. J Neurosci Methods 2008, 167:115-125.

6. Holz EM, Kaufmann T, Desideri L, Malavasi M, Hoogerwerf E-J, Kübler A, Allison BZ, Dunne S, Leeb R, Del R, Millán J, Nijholt A: User Centred Design in BCI Development. In Towards Practical Brain-Computer Interfaces. Berlin, Heidelberg: Springer Berlin Heidelberg; 2012:155-172.

7. Kaufmann T, Schulz SM, Köblitz A, Renner G, Wessig C, Kübler A: Face stimuli effectively prevent brain-computer interface inefficiency in patients with neurodegenerative disease. Clin Neurophysiol 2012. http://dx.doi.org/10.1016/j.clinph.2012.11.006

8. Kübler A, Nijboer F, Mellinger J, Vaughan TM, Pawelzik H, Schalk G, McFarland DJ, Birbaumer N, Wolpaw JR: Patients with ALS can use sensorimotor rhythms to operate a brain-computer interface. Neurology 2005, 64:1775-1777.

9. Nijboer F, Sellers EW, Mellinger J, Jordan MA, Matuz T, Furdea A, Halder S, Mochty U, Krusienski DJ, Vaughan TM, et al: A P300-based brain-computer interface for people with amyotrophic lateral sclerosis. Clin Neurophysiol 2008, 119:1909-1916.

10. Zickler C, Riccio A, Leotta F, Hillian-Tress S, Halder S, Holz E, Staiger-Sälzer P, Hoogerwerf E-J, Desideri L, Mattia D, Kübler A: A brain-computer interface as input channel for a standard assistive technology software. Clin EEG Neurosci 2011, 42:236-244. 
11. Millán JR, Renkens F, Mourino J, Gerstner W: Noninvasive brain-actuated control of a mobile robot by human EEG. IEEE Trans Biomed Eng 2004, 51:1026-1033.

12. Philips J, Del R, Millán J, Vanacker G, Lew E, Galan F, Ferrez PW, Van Brussel $H$, Nuttin M: Adaptive Shared Control of a Brain-Actuated Simulated Wheelchair, IEEE 10th International Conference on Rehabilitation Robotics; ICORR 2007. IEEE; 2007:408-414

13. Brach JS, VanSwearingen J: Measuring fatigue related to facial muscle function. Arch Phys Med Rehabil 1995, 76:905-908.

14. Pfurtscheller $G$, Neuper $C$ : Motor imagery activates primary sensorimotor area in humans. Neurosci Lett 1997, 239:65-68.

15. Pfurtscheller G, Neuper C, Flotzinger D, Pregenzer M: EEG-based discrimination between imagination of right and left hand movement. Electroencephalogr Clin Neurophysiol 1997, 103:642-651.

16. Galán F, Nuttin M, Lew E, Ferrez PW, Vanacker G, Philips J, Millán JR: A brain-actuated wheelchair: asynchronous and non-invasive braincomputer interfaces for continuous control of robots. Clin Neurophysiol 2008, 119:2159-2169.

17. Hema CR, Paulraj MP, Yaacob S, Adom AH, Nagarajan R: Asynchronous brain machine interface-based control of a wheelchair. Adv Exp Med Biol 2011, 696:565-572

18. Leeb R, Friedman D, Müller-Putz GR, Scherer R, Slater M, Pfurtscheller G: Self-paced (asynchronous) $\mathrm{BCl}$ control of a wheelchair in virtual environments: a case study with a tetraplegic. Comput Intell Neurosci 2007, 2007:1-8.

19. Lew E, Nuttin M, Ferrez PW, Degeest R, Buttfield A, Vanacker G, Millán JDR: Noninvasive brain-computer interface for mental control of a simulated wheelchair. In Book Noninvasive brain-computer interface for mental control of a simulated wheelchair. Graz City;Austria: Graz University of Technology; 2006.

20. Huang D, Qian K, Fei D-Y, Jia W, Chen X, Bai O: Electroencephalography (EEG)-based brain-computer interface (BCl): a 2-D virtual wheelchair control based on event-related desynchronization/synchronization and state control. IEEE Trans Neural Syst Rehabil Eng 2012, 20:379-388.

21. Pfurtscheller G, Leeb R, Keinrath C, Friedman D, Neuper C, Guger C, Slater M: Walking from thought. Brain Res 2006, 1071:145-152.

22. Tanaka K, Matsunaga K, Wang HO: Electroencephalogram-Based Control of an Electric Wheelchair. IEEE Trans Robot 2005, 21:762-766.

23. Leeb R, Keinrath C, Friedman D, Guger C, Scherer R, Neuper C, Garau M, Antley A, Steed A, Slater M, Pfurtscheller G: Walking by thinking: the brainwaves are crucial, not the muscles! Presence: Teleoperators and Virtual Environ 2006, 15:500-514

24. Cincotti F, Kauhanen L, Aloise F, Palomaki T, Caporusso N, Jylanki P, Mattia D, Babiloni F, Vanacker G, Nuttin M, et al: Vibrotactile feedback for braincomputer interface operation. Comput Intell Neurosci 2007, 2007:48937. doi:10.1155/2007/48937.

25. Leeb R, Gwak K, Kim DS, Millán JR: Feel the BCI Vibe - Vibrotactile BCl Feedback. Monterey, USA: Fifth International Brain-Computer Interface Meeting 2013; 2013.

26. Carlson T, Monnard G, Millán JR: Vision-based shared control for a $\mathrm{BCl}$ wheelchair. Int J Bioelectromagnetism 2011, 13:20-21

27. Tonin L, Leeb R, Tavella M, Perdikis S, Millán JR: The role of shared-control in BCl-based telepresence, Book The role of shared-control in BCl-based telepresence (Editor ed.^eds.). City: IEEE; 2010:1462-1466. 1462-1466.

28. Vanacker G, Millán JR, Lew E, Ferrez PW, Moles FG, Philips J, Van Brussel H, Nuttin M: Context-based filtering for assisted brain-actuated wheelchair driving. Comput Intell Neurosci 2007, 2007:1-12.

29. Thurlings ME, Erp JBF, Brouwer A-M, Werkhoven PJ: EEG-Based Navigation from a Human Factors Perspective. In Brain-Computer Interfaces. Edited by Tan DS, Nijholt A. London: Springer; 2010:71-86. Human-Computer Interaction Series.

30. Tsui CSL, Gan JQ, Hu H: A self-paced motor imagery based brain-computer interface for robotic wheelchair control. Clin EEG Neurosci 2011, 42:225-229.

31. Blankertz B, Sannelli C, Halder S, Hammer EM, Kübler A, Müller K-R, Curio G, Dickhaus T: Neurophysiological predictor of SMR-based $\mathrm{BCl}$ performance. Neuroimage 2010, 51:1303-1309.

32. Guger C, Daban S, Sellers E, Holzner C, Krausz G, Carabalona R, Gramatica F, Edlinger G: How many people are able to control a P300-based brain-computer interface (BCI)? Neurosci Lett 2009, 462:94-98.

33. Guger C, Edlinger G, Harkam W, Niedermayer I, Pfurtscheller G: How many people are able to operate an EEG-based brain-computer interface $(\mathrm{BCl})$ ? IEEE Trans Neural Syst Rehabil Eng 2003, 11:145-147.

34. Halder S, Agorastos D, Veit R, Hammer EM, Lee S, Varkuti B, Bogdan M, Rosenstiel W, Birbaumer N, Kübler A: Neural mechanisms of brain-computer interface control. Neuroimage 2011, 55:1779-1790.
35. Hammer EM, Halder S, Blankertz B, Sannelli C, Dickhaus T, Kleih S, Müller K-R, Kübler A: Psychological predictors of SMR-BCI performance. Biol Psychol 2012, 89:80-86.

36. Sellers EW, Arbel Y, Donchin E: BCls that use P300 Event-Related Potentials. In Wolpaw and Wolpaw (2012), Brain Computer Interfaces: Principles and Practice. Austria: Graz University of Technology; 2012.

37. Sutton S, Braren M, Zubin J, John ER: Evoked-potential correlates of stimulus uncertainty. Science (New York, NY) 1965, 150:1187-1188.

38. Polich J: Updating P300: an integrative theory of P3a and P3b. Clin Neurophysiol 2007, 118:2128-2148.

39. Farwell LA, Donchin E: Talking off the top of your head: toward a mental prosthesis utilizing event-related brain potentials. Electroencephalogr Clin Neurophysiol 1988, 70:510-523.

40. Kaufmann T, Hammer EM, Kübler A: ERPs contributing to classification in the P300 BCl, Proceedings of the fifth international Brain-Computer Interface Conference. Graz, Austria; 2011:136-139.

41. Fazel-Rezai R, Allison BZ, Guger C, Sellers EW, Kleih SC, Kubler A: P300 brain computer interface: current challenges and emerging trends. Front Neuroeng 2012, 5:14

42. Kleih SC, Kaufmann T, Zickler C, Halder S, Leotta F, Cincotti F, Aloise F, Riccio A, Herbert C, Mattia D, Kübler A: Out of the frying pan into the fire-the P300based BCI faces real-world challenges. Prog Brain Res 2011, 194:27-46.

43. Mak JN, Arbel Y, Minett JW, McCane LM, Yuksel B, Ryan D, Thompson D, Bianchi L, Erdogmus D: Optimizing the P300-based brain-computer interface: current status, limitations and future directions. J Neural Eng 2011, 8:025003.

44. Rebsamen B, Guan C, Zhang H, Wang C, Teo C, Ang MH, Burdet E: A brain controlled wheelchair to navigate in familiar environments. IEEE Trans Neural Syst Rehabil Eng 2010, 18:590-598.

45. Bell CJ, Shenoy P, Chalodhorn R, Rao RPN: Control of a humanoid robot by a noninvasive brain-computer interface in humans. J Neural Eng 2008, 5:214-220.

46. Pires G, Castelo-Branco M, Nunes U: Visual P300-based BCl to steer a wheelchair: a Bayesian approach. Conf Proc IEEE Eng Med Biol Soc 2008, 2008:658-661.

47. Iturrate I, Antelis JM, Kübler A, Minguez J: A noninvasive brain-actuated wheelchair based on a P300 neurophysiological protocol and automated navigation. IEEE Trans Robot 2009, 25:614-627.

48. Escolano C, Antelis JM, Minguez J: A telepresence mobile robot controlled with a noninvasive brain \#x2013; computer interface. IEEE Trans Syst Man Cybern B Cybern 2012, 42:793-804.

49. Long J, Li Y, Wang H, Yu T, Pan J, Li F: A hybrid brain computer interface to control the direction and speed of a simulated or real wheelchair. IEEE Trans Neural Syst Rehabil Eng 2012, 20:720-729.

50. Aloise F, Lasorsa I, Brouwer AM, Mattia D, Babiloni F, Salinari S, Marciani MG, Cincotti F: Multimodal stimulation for a P300-based BCl. Int J Bioelectromagnetism 2007, 9:128-130.

51. Diez PF, Torres Müller SM, Mut VA, Laciar E, Avila E, Bastos-Filho TF, Sarcinelli-Filho $\mathrm{M}$ : Commanding a robotic wheelchair with a high-frequency steady-state visual evoked potential based brain-computer interface. Med Eng Phys 2013, 35(8):1155-1164.

52. Brouwer A-M, Van Erp JBF: A Tactile P300 Brain-Computer Interface. Front Neurosci 2010, 19(4):4

53. Brouwer A-M, Van Erp JBF, Aloise F, Cincotti F: Tactile, visual, and bimodal P300s: could bimodal P300s boost BCI performance? SRX Neuroscience 2010, 2010:1-9.

54. Thurlings ME, Van Erp JBF, Brouwer A-M, Blankertz B, Werkhoven P: Control-display mapping in brain-computer interfaces. Ergonomics 2012, 55:564-580.

55. Kaufmann T, Holz EM, Kübler A: Comparison of tactile, auditory and visual modality for brain-computer interface use: A case study with a patient in the locked-in state. Front Neurosci 2013, 7:129. doi:10.3389/ fnins.2013.00129.

56. Höhne J, Schreuder M, Blankertz B, Tangermann M: Two-dimensional auditory p300 speller with predictive text system. Conf Proc IEEE Eng Med Biol Soc 2010, 2010:4185-4188.

57. Jin J, Allison BZ, Sellers EW, Brunner C, Horki P, Wang X, Neuper C: An adaptive P300-based control system. J Neural Eng 2011, 8:036006.

58. Lenhardt A, Kaper M, Ritter HJ: An adaptive P300-based online braincomputer interface. IEEE Trans Neural Syst Rehabil Eng 2008, 16:121-130.

59. Liu T, Goldberg L, Gao S, Hong B: An online brain-computer interface using non-flashing visual evoked potentials. J Neural Eng 2010, 7:036003.

60. Schreuder M, Rost T, Tangermann M: Listen, you are writing! Speeding up online spelling with a dynamic auditory BCl. Front Neuroprosthetics 2011, 5:112. 
61. Schreuder M, Höhne J, Blankertz B, Haufe S, Dickhaus T, Tangermann M: Optimizing event-related potential based brain-computer interfaces: a systematic evaluation of dynamic stopping methods. J Neural Eng 2013, 10:036025

62. Schreuder M, Höhne J, Treder M, Blankertz B, Tangermann M: Performance optimization of ERP-based $\mathrm{BCl}$ s using dynamic stopping. Conf Proc IEEE Eng Med Biol Soc 2011, 2011:4580-4583.

63. Maguire MC: User-Centred Requirements Handbook WP5 D53 of the Telematics Applications Project TE - RESPECT: Requirements Engineering and Specification in Telematics; 1998

64. Schalk G, McFarland DJ, Hinterberger T, Birbaumer N, Wolpaw JR: BCI2000: A general-purpose, brain-computer interface $(\mathrm{BCl})$ system. IEEE Trans Biomed Eng 2004, 51:1034-1043.

65. Oostenveld R, Fries P, Maris E, Schoffelen J-M: FieldTrip: open source software for advanced analysis of MEG, EEG, and invasive electrophysiological data. Comput Intell Neurosci 2011, 2011:156869.

66. Donchin E: Discriminant analysis in average evoked response studies: the study of single trial data. Electroencephalogr Clin Neurophysiol 1969, 27:311-314.

67. Krusienski DJ, Sellers EW, MCFarland DJ, Vaughan TM, Wolpaw JR: Toward enhanced P300 speller performance. J Neurosci Methods 2008, 167:15-21.

68. Kaufmann T, Völker S, Gunesch L, Kübler A: Spelling is just a click away - a user-centered brain-computer interface including auto-calibration and predictive text entry. Front Neurosci 2012, 6:72.

69. Choi K, Cichocki A: Control of a Wheelchair by Motor Imagery in Real Time. In Intelligent Data Engineering and Automated Learning - IDEAL 2008. Edited by Fyfe C, Kim D, Lee S-Y, Yin H. Berlin Heidelberg: Springer; 2008:330-337. Lecture Notes in Computer Science.

70. Scherer R, Lee F, Schlogl A, Leeb R, Bischof H, Pfurtscheller G: Toward self-paced brain-computer communication: navigation through virtual worlds. IEEE Trans Biomed Eng 2008, 55:675-682.

71. Jin J, Sellers EW, Zhang Y, Daly I, Wang X, Cichocki A: Whether generic model works for rapid ERP-based BCl calibration. J Neurosci Methods 2012, 212:94-99.

72. Lu H, Plataniotis KN, Venetsanopoulos AN: Regularized common spatial patterns with generic learning for EEG signal classification. Conf Proc IEEE Eng Med Biol Soc 2009, 2009:6599-6602.

73. Herweg A, Kaufmann T, Kübler A: Increasing tactile ERP-BCl performance using generic models, 4th Workshop on Tools for Brain Computer Interaction (TOBI); January 17; Sion; 2013

74. Millán JR, Rupp R, Müller-Putz GR, Murray-Smith R, Giugliemma C, Tangermann M, Vidaurre C, Cincotti F, Kübler A, Leeb R, et al: Combining brain-computer interfaces and assistive technologies: state-of-the-art and challenges. Front Neuroprosthetics 2010, 4:161.

75. Zickler C, Di Donna V, Kaiser V, Al-Khodairy A, Kleih S, Kübler A: BCl applications for people with disabilities: defining user needs and user requirements. In Assistive Technology from Adapted Equipment to Inclusive Environments, AAATE 25 Assistive Technology Research Series. Edited by Emiliani PL, Burzagli L, Como A Gabbanini F, Salminen A-L. Amsterdam: IOS Press; 2009:185-189.

76. Kübler A, Holz E, Kaufmann T, Zickler C: A User Centred Approach for Bringing $\mathrm{BCl}$ Controlled Applications to End-Users. In Brain-Compute Interface Systems - Recent Progress and Future Prospects. Edited by Fazel-Rezai R. Rijeka, Croatia: Intech; 2013.

77. Cheliout-Heraut F, Barois A, Urtizberea A, Viollet L, Estournet-Mathiaud B: Evoked potentials in spinal muscular atrophy. J Child Neurol 2003, 18:383-390.

78. Halder S, Baykara E, Fioravanti C, Simon N, Käthner I, Pasqualotto E, Kleih S, Ruf CA, Birbaumer N, Kübler A: Training Effects of Multiple Auditory BCI Sessions, Fifth International Brain-Computer Interface Meeting 2013. Monterey, USA; 2013.

79. Kaufmann T, Schulz SM, Grünzinger C, Kübler A: Flashing characters with famous faces improves ERP-based brain-computer interface performance. J Neural Eng 2011, 8:056016.

80. Aloise F, Schettini F, Aricò P, Leotta F, Salinari S, Mattia D, Babiloni F, Cincotti F: P300-based brain-computer interface for environmental control: an asynchronous approach. J Neural Eng 2011, 8:025025.

81. Aloise F, Schettini F, Aricò P, Salinari S, Guger C, Rinsma J, Aiello M, Mattia D. Cincotti F: Asynchronous P300-based brain-computer interface to control a virtual environment: initial tests on end users. Clin EEG Neurosci 2011, 42:219-224.

82. Zhang H, Guan C, Wang C: Asynchronous P300-based brain-computer interfaces: a computational approach with statistical models. IEEE Trans Biomed Eng 2008, 55:1754-1763.
83. Allison B, Leeb R, Brunner C, Müller-Putz G, Bauernfeind G, Kelly J, Neuper C: Toward smarter $\mathrm{BCls}$ : extending $\mathrm{BCl}$ s through hybridization and intelligent control. J Neural Eng 2012, 9:013001.

84. Brunner C, Allison B, Altstätter C, Neuper C: A comparison of three braincomputer interfaces based on event-related desynchronization, steady state visual evoked potentials, or a hybrid approach using both signals. J Neural Eng 2011, 8:025010.

85. Holz E, Riccio A, Reichert J, Leotta F, Aricò P, Cincotti F, Mattia D, Kübler A: HybridP300 BCl: Usability Testing by Severely Motor-restricted End-Users, 4th Workshop on Tools for Brain Computer Interaction (TOBI). Sion, Switzerland; 2013:111-112.

86. Pfurtscheller G, Allison BZ, Brunner C, Bauernfeind G, Solis-Escalante T, Scherer R, Zander TO, Mueller-Putz G, Neuper C, Birbaumer N: The hybrid $\mathrm{BCl}$. Front Neurosci 2010, 4(4):4.

doi:10.1186/1743-0003-11-7

Cite this article as: Kaufmann et al:: Toward brain-computer interface based wheelchair control utilizing tactually-evoked event-related potentials. Journal of NeuroEngineering and Rehabilitation 2014 11:7.

\section{Submit your next manuscript to BioMed Central and take full advantage of:}

- Convenient online submission

- Thorough peer review

- No space constraints or color figure charges

- Immediate publication on acceptance

- Inclusion in PubMed, CAS, Scopus and Google Scholar

- Research which is freely available for redistribution

Submit your manuscript at www.biomedcentral.com/submit
C) Biomed Central 